\title{
Stability of rectangular cantilever plates with high elasticity
}

\author{
Mikhail Sukhoterin ${ }^{1,}$, Sergey Baryshnikov ${ }^{1}$, Tatiana Knysh $^{1}$, and Elena Rasputina ${ }^{1}$ \\ ${ }^{1}$ Admiral Makarov State University of Maritime and Inland Shipping, Saint Petersburg, Russia
}

\begin{abstract}
The problem of cantilever plate stability has been little studied due to the difficulty of solving the corresponding boundary problem. The known approximate solutions mainly concern only the first critical load. In this paper, stability of an elastic rectangular cantilever plate under the action of uniform pressure applied to its edge opposite to the clamped edge is investigated. Under such conditions, thin canopies of buildings made of new materials can be found at sharp gusts of wind in longitudinal direction. At present, cantilever nanoplates are widely used as key components of sensors to create nanoscale transistors where they are exposed to magnetic fields in the plate plane. The aim of the study is to obtain the critical force spectrum and corresponding forms of supercritical equilibrium. The deflection function is selected as a sum of two hyperbolic trigonometric series with adding special compensating summands to the main symmetric solution for the free terms of the decomposition of the functions in the Fourier series by cosines. The fulfillment of all conditions of the boundary problem leads to an infinite homogeneous system of linear algebraic equations with regard to unknown series coefficients. The task of the study is to create a numerical algorithm that allows finding eigenvalues of the resolving system with high accuracy. The search for critical loads (eigenvalues) giving a nontrivial solution of this system is carried out by brute force search of compressive load value in combination with the method of sequential approximations. For the plates with different side ratios, the spectrum of the first three critical loads is obtained, at which new forms of equilibrium emerge. An antisymmetric solution is obtained and studied. 3D images of the corresponding forms are presented.
\end{abstract}

\section{Introduction}

The classical problem of stability of a thin rectangular cantilever plate under the action of only compressive pressure applied to the free edge, parallel to the clamped edge, has no precise solution in a closed form. This problem on eigenvalues is similar to the problem of determination of the spectrum of resonance frequencies and forms of oscillations of the cantilever plate $[1,2]$.

It is assumed that the plate material is perfectly elastic, so there is an infinite number of critical loads that change the form of equilibrium of the plate. In practice, usually only the first critical load is calculated, which is considered destructive, but the plates from new

* Corresponding author: sukhoterinmv@gumrf.ru 
high-elastic materials in the presence of constructive limiters of their bending can acquire other forms of equilibrium with an increasing load, including asymmetric. Therefore, the following one or two critical loads and corresponding forms of stability loss are also of practical interest.

The problem of plate stability using various methods of its solution is discussed in works [3-10]. In [11-16], for the solution of the stability problem of a rectangular plate clamped along the contour, Fourier series were used, which led to an infinite system of linear algebraic equations relative to the series coefficients.

Stability of cantilever plates has been little studied. The complexity of the corresponding boundary problem of mathematical physics requires the development of reliable approximate methods of its solution. Practical results are obtained, perhaps, only in [17-23].

In $[17,18]$, various types of a compressive load on the plate contour were considered. In the first of them, the distributed compressive load is applied only to the edge parallel to the clamped edge, while in the second one, only the edges perpendicular to the clamped edge were compressed. The first critical loads were found using a variation method. In [19], the compressive load is applied only to one edge perpendicular to the clamped edge. To find a solution, the condition of total energy minimum of the plate was used. A similar problem was solved in work [20], in which the approximating function was selected as a polynomial. The case of compressive load on all edges was not considered.

In [21], using the finite element method (FEM), the first critical loads for various cases of concentrated compressive forces were found.

The stability of a cantilever oblique heterogeneous plate in a supersonic gas flow was investigated in [22], where the critical numbers were found by FEM using Hamilton's variational principle.

Paper [23] deals with cantilever nanoplates that are exposed to magnetic fields in their plane. The analytical solution is constructed within the framework of the nonlocal theory of elasticity of Eringen [24] by the simplex method using trigonometric series.

It should be noted that the wide application of numerical methods, such as FEM, FDM (finite difference method), etc., faces the problem of verification of boundary conditions. Such verification is very difficult because these methods work with arrays of numbers, rather than with analytical expressions that can be substituted in boundary conditions.

In the present work, the exact solution of the problem is found using hyperbolic trigonometric series. The problem was reduced to solving an infinite homogeneous system of linear algebraic equations for the coefficients of these series. The nontrivial values of the coefficients can be obtained by equating the system determinant to zero. However, making a determinant and solving the corresponding equation which gives a spectrum of critical loads is a very laborious process. Here, the method of brute force search of the load parameter in combination with the iterative method of coefficients determination is proposed. For a given load, the initial values of the coefficients of the first series are set arbitrarily as a descending sequence. These values and the values of the other coefficients are specified in the course of the iteration process, which at this load value will either converge to the trivial solutions for the coefficients (stable equilibrium), or to the nontrivial solutions (new form of equilibrium), or the process will diverge, defining the instability interval for the compressive loads. In the latter case, the non-linear theory should be used for supercritical state analysis. 


\section{Methods}

Let the uniform compressive forces with intensity $T_{y}$, acting in the plane of a thin homogeneous rectangular cantilever plate of constant thickness, be applied to its free edge $y$ $=1$ (Fig.1).

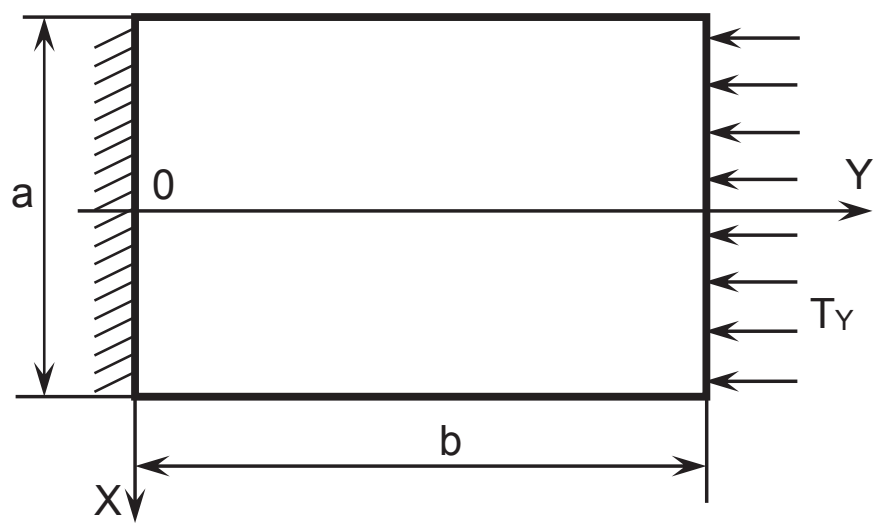

Fig. 1. Cantilever plate under the action of uniform pressure on its edge $y=1$. [25]

The differential equation of bending of the plate in a stable state has the following form

$$
D \nabla^{2} \nabla^{2} W+T_{Y} \frac{\partial^{2} W}{\partial Y^{2}}=0
$$

where $W$ is the deflection of the plate's middle surface, $D=E h^{3} /\left[12\left(1-v^{2}\right)\right]$ is the cylindrical stiffness of the plate, $E$ is Young's modulus, $h$ is the plate's thickness, $v$ is Poisson's ratio, $\nabla^{2} \nabla^{2}=\frac{\partial^{4}}{\partial x^{4}}+2 \frac{\partial^{4}}{\partial x^{2} \partial y^{2}}+\frac{\partial^{4}}{\partial y^{4}}$ is the biharmonic operator, $X, Y$ are coordinates of the plate's points.

This equation always has a trivial solution, which corresponds to the basic flat form of the plate's equilibrium. When the critical value of the compressive load is reached, this form ceases to be the only one and another form of equilibrium may appear, corresponding to the minimum potential energy of the plate under this load. The plate loses stability of the flat form and moves to the new form of equilibrium.

Let us move on to dimensionless coordinates $x=X / b, y=Y / b$. Then, equation (1) will be written down as follows:

$$
\nabla^{2} \nabla^{2} w+T_{y} \frac{\partial^{2} w}{\partial y^{2}}=0,
$$

where $w=W / b$ is the relative deflection, $T_{y}=T_{Y} b^{2} / D$ is the intensity of relative compressive forces. The relative dimensions of the plate will be as follows: $-\gamma / 2 \leq x \leq \gamma / 2, \quad 0 \leq y \leq 1$

where $\gamma=a / b$.

Boundary conditions [25, 23]: 


$$
\begin{aligned}
& w=0, \quad \frac{\partial w}{\partial y}=0, \quad \text { on edge } y=0 \\
& \frac{\partial^{2} w}{\partial y^{2}}+v \frac{\partial^{2} w}{\partial x^{2}}=0, \quad \frac{\partial^{3} w}{\partial y^{3}}+(2-v) \frac{\partial^{3} w}{\partial x^{2} \partial y}+T_{y} \frac{\partial w}{\partial y}=0, \quad \text { on edge } y=1 \\
& \frac{\partial^{2} w}{\partial x^{2}}+v \frac{\partial^{2} w}{\partial y^{2}}=0, \quad \frac{\partial^{3} w}{\partial x^{3}}+(2-v) \frac{\partial^{3} w}{\partial x \partial y^{2}}=0, \quad \text { on edges } x= \pm \gamma / 2 \\
& \frac{\partial^{2} w}{\partial x \partial y}=0 . \quad \text { at points }( \pm \gamma / 2,1)
\end{aligned}
$$

Here, equations (3) are geometric conditions of rigid restraint. They express the absence of deflections and angles of rotation of edge $y=0$. Equations $(4,5)$ are the conditions of absence of bending moments and cutting forces on the free edges. Condition (6) excludes torques at the angular points of the free part of the contour.

The problem (2-6) always has a trivial (zero) solution for the deflection function. This corresponds to the stable non-deformed state of the plate. In addition to the non-trivial solution, the problem may have non-trivial solutions at certain values of load $T y$ when after the loss of stability, the plate acquires a new form of equilibrium corresponding to the minimum potential energy of the plate.

It should be noted that, despite the symmetry of the problem, the equilibrium forms can be both symmetric relative to axis Oy and antisymmetric.

The method proposed in this paper is based on the use of hyperbolic trigonometric series with undefined coefficients to solve all equations of the problem. This leads to an infinite system of linear algebraic equations for the coefficients of the series, containing the intensity of the compressive load as a parameter. The nontrivial solutions of the system giving the spectrum of critical loads are determined by the simple brute force search of the load value in combination with the method of sequential approximations to correct arbitrary initial values of the coefficients.

\subsection{Symmetrical solution}

The desired symmetric solution will be chosen as a superposition of two hyperbolic trigonometric series

$$
\begin{gathered}
w_{1}(x, y)=\sum_{k=1,3, \ldots}^{\infty}\left(A_{k} \cosh \alpha_{k} x+B_{k} \cosh \beta_{k} x\right) \sin \lambda_{k} y \\
w_{2}(x, y)=\sum_{s=1}^{\infty}(-1)^{s}\left(C_{s} \sinh \xi_{s} \tilde{y}+D_{s} \sinh \eta_{s} \tilde{y}+E_{s} \cosh \xi_{s} \tilde{y}+F_{s} \cosh \eta_{s} \tilde{y}\right) \cos \mu_{s} x,
\end{gathered}
$$

where $A_{k}, B_{k}, C_{s}, D_{s}, E_{s}, F_{s}, \alpha_{k}, \beta_{k}, \xi_{s}, \eta_{s}$ are the undefined coefficients, $\lambda_{k}=k \pi / 2 ; \mu_{s}$ $=2 \pi s / \gamma, \tilde{y}=y-1$. Let us note that both these functions "automatically" meet boundary condition (6).

Let us ask for each of them to satisfy basic differential equation (2). Then, for coefficients $\alpha_{k}, \beta_{k}, \xi_{s}, \eta_{s}$ we will obtain biquadratic equations

$$
\alpha_{k}^{4}-2 \alpha_{k}^{2} \lambda_{k}^{2}+\lambda_{k}^{4}-T_{y} \lambda_{k}^{2}=0, \xi_{s}^{4}-2 \xi_{s}^{2} \mu_{s}^{2}+\mu_{s}^{4}+T_{y} \xi_{s}^{2}=0,
$$




$$
\beta_{k}^{4}-2 \beta_{k}^{2} \lambda_{k}^{2}+\lambda_{k}^{4}-T_{y} \lambda_{k}^{2}=0, \eta_{s}^{4}-2 \eta_{s}^{2} \mu_{s}^{2}+\mu_{s}^{4}+T_{y} \xi_{s}^{2}=0,
$$

which have four roots each, however, relying on the properties of hyperbolic functions, it is enough to take a pair of roots from the quartets:

$$
\begin{gathered}
\alpha_{k}=\sqrt{\lambda_{k}^{2}+\lambda_{k} \sqrt{T_{y}}}, \quad \beta_{k}=\sqrt{\lambda_{k}^{2}-\lambda_{k} \sqrt{T_{y}}} \\
\xi_{s}=\sqrt{0.5\left(2 \mu_{s}^{2}-T_{y}+\sqrt{T_{y}^{2}-4 \mu_{s}^{2} T_{y}}\right)}, \quad \eta_{s}=\sqrt{0.5\left(2 \mu_{s}^{2}-T_{y}-\sqrt{T_{y}^{2}-4 \mu_{s}^{2} T_{y}}\right)} .
\end{gathered}
$$

Let us now ask for the sum of functions $(7,8)$ to meet boundary conditions $(3-5)$. Then we get a system of equations

$$
\begin{aligned}
& \sum_{s=1}^{\infty}(-1)^{s}\left(-C_{s} \sinh \xi_{s}-D_{s} \sinh \eta_{s}+E_{s} \cosh \xi_{s}+F_{s} \cosh \eta_{s}\right) \cos \mu_{s} x=0 \\
& \sum_{s=1}^{\infty}(-1)^{s}\left(\begin{array}{l}
C_{s} \xi_{s} \cosh \xi_{s}+D_{s} \eta_{s} \cosh \eta_{s} \\
-E_{s} \xi_{s} \sinh \xi_{s}-F_{s} \eta_{s} \sinh \eta_{s}
\end{array}\right) \cos \mu_{s} x+\sum_{k=1,3, \ldots}^{\infty} \lambda_{k}\left(A_{k} \cosh \alpha_{k} x+B_{k} \cosh \beta_{k} x\right)=0 \\
& \sum_{s=1}^{\infty}(-1)^{s}\left[E_{s}\left(\xi_{s}^{2}-v \mu_{s}^{2}\right)+F_{s}\left(\eta_{s}^{2}-v \mu_{s}^{2}\right)\right] \cos \mu_{s} x-\sum_{k=1,3, \ldots}^{\infty}(-1)^{\tilde{k}}\left[\begin{array}{l}
A_{k}\left(v \alpha_{k}^{2}-\lambda_{k}^{2}\right) \cosh \alpha_{k} x \\
+B_{k}\left(v \beta_{k}^{2}-\lambda_{k}^{2}\right) \cosh \beta_{k} x
\end{array}\right]=0 \\
& \sum_{s=1}^{\infty}(-1)^{s}\left\{C_{s} \xi_{s}\left[\xi_{s}^{2}-(2-v) \mu_{s}^{2}+T_{y}\right]+D_{s} \eta_{s}\left[\eta_{s}^{2}-(2-v) \mu_{s}^{2}+T_{y}\right]\right\} \cos \mu_{s} x=0, \\
& -\sum_{s=1}^{\infty}\left[\begin{array}{c}
C_{s}\left(\mu_{s}^{2}-v \xi_{s}^{2}\right) \sinh \xi_{s} \tilde{y} \\
+D_{s}\left(\mu_{s}^{2}-v \eta_{s}^{2}\right) \sinh \eta_{s} \tilde{y} \\
+E_{s}\left(\mu_{s}^{2}-v \xi_{s}^{2}\right) \cosh \xi_{s} \tilde{y} \\
+F_{s}\left(\mu_{s}^{2}-v \eta_{s}^{2}\right) \cosh \eta_{s} \tilde{y}
\end{array}\right]+\sum_{k=1,3, \ldots[}^{\infty}\left[\begin{array}{c}
A_{k}\left(\alpha_{k}^{2}-v \lambda_{k}^{2}\right) \cosh \tilde{\alpha}_{k} \\
+B_{k}\left(\beta_{k}^{2}-v \lambda_{k}^{2}\right) \cosh \tilde{\beta}_{k}
\end{array}\right] \sin \lambda_{k} y=0, \\
& \sum_{k=1,3, \ldots}^{\infty}\left\{A_{k} \alpha_{k}\left[\alpha_{k}^{2}-(2-v) \lambda_{k}^{2}\right] \sinh \tilde{\alpha}_{k}+B_{k} \beta_{k}\left[\beta_{k}^{2}-(2-v) \lambda_{k}^{2}\right] \sinh \tilde{\beta}_{k}\right\} \sin \lambda_{k} y=0 .
\end{aligned}
$$

Here $\tilde{\alpha}_{k}=\alpha_{k} \gamma / 2, \tilde{\beta}_{k}=\beta_{k} \gamma / 2, \tilde{k}=(k+1) / 2$.

From this system, it is necessary to obtain a system of linear algebraic equations relative to coefficients $A_{k}, B_{k}, C_{s}, D_{s}, E_{s}, F_{s}$. Since in equations (12), (13), (15), the summation in the series is carried out by different indexes, then hyperbolic functions in them will be decomposed into Fourier series.

In equations (12) and (13) we use the known decomposition of hyperbolic functions into Fourier series by $\cos \mu_{s} x$

$$
\begin{array}{r}
\cosh \alpha_{k} x=\sinh \tilde{\alpha}_{k}\left[\frac{1}{\tilde{\alpha}_{k}}+\frac{4 \alpha_{k}}{\gamma} \sum_{s=1}^{\infty} \frac{(-1)^{s}}{\alpha_{k}^{2}+\mu_{s}^{2}} \cos \mu_{s} x\right] \\
\cosh \beta_{k} x=\sinh \tilde{\beta}_{k}\left[\frac{1}{\tilde{\beta}_{k}}+\frac{4 \beta_{k}}{\gamma} \sum_{s=1}^{\infty} \frac{(-1)^{s}}{\beta_{k}^{2}+\mu_{s}^{2}} \cos \mu_{s} x\right],
\end{array}
$$


then these equations will take the following form:

$$
\begin{aligned}
\sum_{s=1}^{\infty}(-1)^{s} & \left(\begin{array}{l}
C_{s} \xi_{s} \cosh \xi_{s}+D_{s} \eta_{s} \cosh \eta_{s} \\
-E_{s} \xi_{s} \sinh \xi_{s}-F_{s} \eta_{s} \sinh \eta_{s}
\end{array}\right) \cos \mu_{s} x+\sum_{k=1,3, \ldots}^{\infty} \lambda_{k}\left(A_{k} \frac{\sinh \tilde{\alpha}_{k}}{\tilde{\alpha}_{k}}+B_{k} \frac{\sinh \tilde{\beta}_{k}}{\tilde{\beta}_{k}}\right) \\
& +\frac{4}{\gamma} \sum_{s=1}^{\infty}(-1)^{s} \sum_{k=1,3, \ldots}^{\infty} \lambda_{k}\left(A_{k} \frac{\alpha_{k} \sinh \tilde{\alpha}_{k}}{\alpha_{k}^{2}+\mu_{s}^{2}}+B_{k} \frac{\beta_{k} \sinh \tilde{\beta}_{k}}{\beta_{k}^{2}+\mu_{s}^{2}}\right) \cos \mu_{s} x=0 \\
\sum_{s=1}^{\infty}(-1)^{s}\left[E_{s}\left(\xi_{s}^{2}-v \mu_{s}^{2}\right)+F_{s}\left(\eta_{s}^{2}-v \mu_{s}^{2}\right)\right] \cos \mu_{s} x-\sum_{k=1,3, \ldots}^{\infty}(-1)^{\tilde{k}} & {\left[A_{k}\left(v \alpha_{k}^{2}-\lambda_{k}^{2}\right) \frac{\sinh \tilde{\alpha}_{k}}{\tilde{\alpha}_{k}}\right.} \\
& -\frac{4}{\gamma} \sum_{s=1}^{\infty}(-1)^{s} \sum_{k=1,3, \ldots}^{\infty}(-1)^{\tilde{k}}\left[\begin{array}{l}
\left.A_{k}\left(v \beta_{k}^{2}-\lambda_{k}^{2}\right) \frac{\sinh \tilde{\beta}_{k}}{\tilde{\beta}_{k}}\right] \\
+A_{k}\left(v \alpha_{k}^{2}-\lambda_{k}^{2}\right) \frac{\alpha_{k} \sinh \tilde{\alpha}_{k}}{\alpha_{k}^{2}+\mu_{s}^{2}}
\end{array}\right] \cos \mu_{s}^{2} x=0 .
\end{aligned}
$$

Here, in two-fold series, the summation signs are rearranged.

In equation (15), we use decomposition of hyperbolic functions into Fourier series by $\sin \lambda_{k} y$

$\sinh \xi_{s} \tilde{y}=-2 \sum_{k=1,3, \ldots}^{\infty} \frac{(-1)^{\tilde{k}} \xi_{s}+\lambda_{k} \sinh \xi_{s}}{\lambda_{k}^{2}+\xi_{s}^{2}} \sin \lambda_{k} y, \quad \sinh \eta_{s} \tilde{y}=-2 \sum_{k=1,3, \ldots}^{\infty} \frac{(-1)^{\tilde{k}} \eta_{s}+\lambda_{k} \sinh \eta_{s}}{\lambda_{k}^{2}+\eta_{s}^{2}} \sin \lambda_{k} y$ $\cosh \xi_{s} \tilde{y}=2 \cosh \xi_{s} \sum_{k=1,3, . .}^{\infty} \frac{\lambda_{k}}{\lambda_{k}^{2}+\xi_{s}^{2}} \sin \lambda_{k} y, \cosh \eta_{s} \tilde{y}=2 \cosh \eta_{s} \sum_{k=1,3, . .}^{\infty} \frac{\lambda_{k}}{\lambda_{k}^{2}+\eta_{s}^{2}} \sin \lambda_{k} y$

and rearrange the summation marks in the resulting two-fold series:

$$
\begin{aligned}
& \sum_{k=1,3, \ldots}^{\infty}\left[A_{k}\left(\alpha_{k}^{2}-v \lambda_{k}^{2}\right) \cosh \tilde{\alpha}_{k}+B_{k}\left(\beta_{k}^{2}-v \lambda_{k}^{2}\right) \cosh \tilde{\beta}_{k}\right] \sin \lambda_{k} y \\
& +2 \sum_{k=1,3, \ldots}^{\infty} \sum_{s=1}^{\infty}\left[\begin{array}{l}
\frac{\mu_{s}^{2}-v \xi_{s}^{2}}{\lambda_{k}^{2}+\xi_{s}^{2}}\left\{C_{s}\left[(-1)^{\tilde{k}} \xi_{s}+\lambda_{k} \sinh \xi_{s}\right]-E_{s} \lambda_{k} \cosh \xi_{s}\right\} \\
+\frac{\mu_{s}^{2}-v \eta_{s}^{2}}{\lambda_{k}^{2}+\eta_{s}^{2}}\left\{D_{s}\left[(-1)^{\tilde{k}} \eta_{s}+\lambda_{k} \sinh \eta_{s}\right]-F_{s} \lambda_{k} \cosh \eta_{s}\right\}
\end{array}\right] \sin \lambda_{k} y=0 .
\end{aligned}
$$

Since in equations (18), (19), there appeared free terms from decomposition into series by cosines, which we will designate $\varphi_{0}$ and $m_{0}$ respectively, in order to obtain a system of linear equations relative to the coefficients of series (7), (8), it is necessary to introduce an auxiliary deflection function $w_{3}$ which would compensate them, satisfying all conditions of the problem together with the basic solution. This function shall be selected in the form of

$$
w_{3}(y)=\sum_{k=1,3, \ldots}^{\infty} g_{k} \sin \lambda_{k} y+\frac{1}{2} M y^{2}-\Phi y
$$

where coefficients $g_{k}, M, \Phi$ are determined from conditions (2-4) 


$$
\sum_{k=1,3, \ldots}^{\infty} g_{k} \lambda_{k}^{2}\left(\lambda_{k}^{2}-T_{y}\right) \sin \lambda_{k} y+M \cdot T_{y}=0, \sum_{k=1,3, \ldots}^{\infty} g_{k} \lambda_{k}-\Phi+\varphi_{0}=0, \sum_{k=1,3, \ldots}^{\infty}(-1)^{\hat{k}} \lambda_{k}^{2} g_{k}+M-m_{0}=0
$$

Let us expand constant $M \cdot T_{y}$ of the first equation (23) into a Fourier series by sines:

$$
M \cdot T_{y}=M \cdot T_{y} \sum_{k=1,3, \ldots}^{\infty} \frac{1}{\lambda_{k}} \sin \lambda_{k} y .
$$

Then we obtain

$$
g_{k}=-\frac{2 M \cdot T_{y}}{\lambda_{k}^{3}\left(\lambda_{k}^{2}-T_{y}\right)}, \quad M=\frac{m_{0}}{1-2 T_{y} \sum_{k=1,3, \ldots}^{\infty} \frac{(-1)^{\tilde{k}}}{\lambda_{k}\left(\lambda_{k}^{2}-T_{y}\right)}}, \quad \Phi=\varphi_{0}+\sum_{k=1,3, \ldots}^{\infty} g_{k} \lambda_{k} .
$$
(5))

Residual from function $w_{3}$ by bending moment $M_{x}$ on edges $x= \pm \gamma / 2$ (first condition

$$
v\left(M-\sum_{k=1,3, \ldots}^{\infty} g_{k} \lambda_{k}^{2} \sin \lambda_{k} y\right)=2 v M \sum_{k=1,3, \ldots}^{\infty} \frac{\lambda_{k}}{\lambda_{k}^{2}-T_{y}} \sin \lambda_{k} y
$$

will be added to equation (21).

Then the system of equations (11-16), after exclusion of external summation marks taking into consideration formulae (18), (19), (21), (25), (26) will take the final form

$$
\begin{aligned}
& C_{s} \sinh \xi_{s}+D_{s} \sinh \eta_{s}-E_{s} \cosh \xi_{s}-F_{s} \cosh \eta_{s}=0, \\
& C_{s} \xi_{s} \cosh \xi_{s}+D_{s} \eta_{s} \cosh \eta_{s}-E_{s} \xi_{s} \sinh \xi_{s}-F_{s} \eta_{s} \sinh \eta_{s} \\
& +\frac{4}{\gamma} \sum_{k=1,3, \ldots}^{\infty} \lambda_{k}\left(A_{k} \frac{\alpha_{k} \sinh \tilde{\alpha}_{k}}{\alpha_{k}^{2}+\mu_{s}^{2}}+B_{k} \frac{\beta_{k} \sinh \tilde{\beta}_{k}}{\beta_{k}^{2}+\mu_{s}^{2}}\right)=0, \\
& E_{s}\left(\xi_{s}^{2}-v \mu_{s}^{2}\right)+F_{s}\left(\eta_{s}^{2}-v \mu_{s}^{2}\right)-\frac{4}{\gamma} \sum_{k=1,3, \ldots}^{\infty}(-1)^{\tilde{k}}\left[\begin{array}{c}
A_{k}\left(v \alpha_{k}^{2}-\lambda_{k}^{2}\right) \frac{\alpha_{k} \sinh \tilde{\alpha}_{k}}{\alpha_{k}^{2}+\mu_{s}^{2}} \\
+B_{k}\left(v \beta_{k}^{2}-\lambda_{k}^{2}\right) \frac{\beta_{k} \sinh \tilde{\beta}_{k}}{\beta_{k}^{2}+\mu_{s}^{2}}
\end{array}\right]=0, \\
& C_{s} \xi_{s}\left[\xi_{s}^{2}-(2-v) \mu_{s}^{2}+T_{y}\right]+D_{s} \eta_{s}\left[\eta_{s}^{2}-(2-v) \mu_{s}^{2}+T_{y}\right]=0 \text {, } \\
& A_{k}\left(\alpha_{k}^{2}-v \lambda_{k}^{2}\right) \cosh \tilde{\alpha}_{k}+B_{k}\left(\beta_{k}^{2}-v \lambda_{k}^{2}\right) \cosh \tilde{\beta}_{k}= \\
& -2 \sum_{s=1}^{\infty}\left[\begin{array}{l}
\frac{\mu_{s}^{2}-v \xi_{s}^{2}}{\lambda_{k}^{2}+\xi_{s}^{2}}\left\{C_{s}\left[(-1)^{\tilde{k}} \xi_{s}+\lambda_{k} \sinh \xi_{s}\right]-E_{s} \lambda_{k} \cosh \xi_{s}\right\} \\
+\frac{\mu_{s}^{2}-v \eta_{s}^{2}}{\lambda_{k}^{2}+\eta_{s}^{2}}\left\{D_{s}\left[(-1)^{\tilde{k}} \eta_{s}+\lambda_{k} \sinh \eta_{s}\right]-F_{s} \lambda_{k} \cosh \eta_{s}\right\}
\end{array}\right] \\
& -p_{k} \sum_{k=1,3, \ldots}^{\infty}(-1)^{\tilde{k}}\left[\begin{array}{l}
A_{k}\left(v \alpha_{k}^{2}-\lambda_{k}^{2}\right) \frac{\sinh \tilde{\alpha}_{k}}{\tilde{\alpha}_{k}} \\
+B_{k}\left(v \beta_{k}^{2}-\lambda_{k}^{2}\right) \frac{\sinh \tilde{\beta}_{k}}{\tilde{\beta}_{k}}
\end{array}\right] \text {, }
\end{aligned}
$$




$$
A_{k} \alpha_{k}\left[\alpha_{k}^{2}-(2-v) \lambda_{k}^{2}\right] \sinh \tilde{\alpha}_{k}+B_{k} \beta_{k}\left[\beta_{k}^{2}-(2-v) \lambda_{k}^{2}\right] \sinh \tilde{\beta}_{k}=0
$$

where

$$
p_{k}=\frac{2 v \lambda_{k}}{\left(\lambda_{k}^{2}-T_{y}\right)\left(1-2 T_{y} \sum_{k=1,3, \ldots}^{\infty} \frac{(-1)^{\tilde{k}}}{\lambda_{k}\left(\lambda_{k}^{2}-T_{y}\right)}\right)} .
$$

The obtained system of equations (27-32) is an infinite homogeneous system of linear algebraic equations in relation to coefficients $A_{k}, B_{k}, C_{s}, D_{s}, E_{s}, F_{s}$. It always has a trivial solution. However, if the system determinant is equal to zero at some values of parameters of load $T_{y}$, we will obtain nontrivial solutions as well. At these (critical) values of load the plate will take a new form of equilibrium. To present a homogeneous system in a standard form, to compose and reveal the corresponding system determinant, and to find its roots is a very difficult task, therefore, the method of brute force search of parameter $T_{y}$ in combination with the method of successive approximations of determining coefficients $A_{k}, B_{k}, C_{s}, D_{s}, E_{s}, F_{s}$ is proposed here.

To organize the iteration process, the resolving system is divided into two subsystems: (27-30), in which the main coefficients are considered to be $C_{s}, D_{s}, E_{s}, F_{s}$, and (31-32), in which the main coefficients are $A_{k}, B_{k}$ in the left side of the equation. First, the entire right side of equation (31) is substituted with an initial arbitrary descending sequence (in this case $1 / \lambda_{\mathrm{k}}$ ), then a subsystem of equations (31)-(32) is solved with a selected value of compressive load $T_{y}$. The found initial coefficients $A_{k 0}, B_{k 0}$ are substituted in subsystem (27) - (30) from which coefficients $C_{s 0}, D_{s 0}, E_{s 0}, F_{s 0}$ are found, which are then used together with $A_{k 0}, B_{k 0}$ to form the right part of equation (31) and a new solution for $A_{k 1}$, $B_{k 1}$ of system (31-32). Then coefficients $C_{s 1}, D_{s 1}, E_{s 1}, F_{s 1}$ of the first approximation are calculated, and then there is an iteration process for specification of the main coefficients of the problem.

If, at the selected load value, starting with some iteration, the corresponding series coefficients practically coincide in absolute value (up to 4-5 significant digits), then this will be a nontrivial solution of homogeneous system (27)-(32): its determinant will be equal to zero. The corresponding load will determine the new form of equilibrium (minimum potential energy of the plate) after the loss of stability. This load is called the critical load. The load values at which the iteration process leads to a trivial solution of system (27)-(32) will determine the zone of stable equilibrium. Those load values, at which the series coefficients and, consequently, the deflections, will tend to infinity, will determine the zone of unstable state, it is necessary to use the nonlinear theory for the study.

\subsection{Antisymmetric solution}

We will also look for this solution as a sum of two hyperbolic trigonometric series, in which odd functions by variable $x$ will be involved:

$$
\begin{gathered}
w_{1}(x, y)=\sum_{k=1,3, \ldots}^{\infty}\left(A_{k} \sinh \alpha_{k} x+B_{k} \sinh \beta_{k} x\right) \sin \lambda_{k} y \\
w_{2}(x, y)=\sum_{s=1,3, \ldots}^{\infty}(-1)^{\tilde{s}}\left(C_{s} \sinh \xi_{s} \tilde{y}+D_{s} \sinh \eta_{s} \tilde{y}+E_{s} \cosh \xi_{s} \tilde{y}+F_{s} \cosh \eta_{s} \tilde{y}\right) \sin \mu_{s} x
\end{gathered}
$$


In these expressions $A_{k}, B_{k}, C_{s}, D_{s}, E_{s}, F_{s}$ are the new undefined coefficients; $\check{s}=$ $(s+1) / 2$, and the value of $\mu_{s}=\pi s / \gamma$ is also different. Coefficients $\lambda_{k}, \alpha_{k}, \beta_{k}, \xi_{s}, \eta_{s}$ retained their previous values.

Meeting all the boundary conditions of the problem, we will proceed to a system similar to (27)-(32) with the only difference that the last two equations will change (sines with cosines will be swapped):

The resolving system of linear algebraic equations will take the following form:

$$
\begin{gathered}
C_{s} \sinh \xi_{s}+D_{s} \sinh \eta_{s}-E_{s} \cosh \xi_{s}-F_{s} \cosh \eta_{s}=0 \\
C_{s} \xi_{s} \cosh \xi_{s}+D_{s} \eta_{s} \cosh \eta_{s}-E_{s} \xi_{s} \sinh \xi_{s}-F_{s} \eta_{s} \sinh \eta_{s} \\
-\frac{4}{\gamma} \sum_{k=1,3, \ldots}^{\infty} \lambda_{k}\left(A_{k} \frac{\alpha_{k} \cosh \tilde{\alpha}_{k}}{\alpha_{k}^{2}+\mu_{s}^{2}}+B_{k} \frac{\beta_{k} \cosh \tilde{\beta}_{k}}{\beta_{k}^{2}+\mu_{s}^{2}}\right)=0 \\
E_{s}\left(\xi_{s}^{2}-v \mu_{s}^{2}\right)+F_{s}\left(\eta_{s}^{2}-v \mu_{s}^{2}\right) \\
+\frac{4}{\gamma} \sum_{k=1,3, \ldots}^{\infty}(-1)^{\tilde{k}}\left[A_{k}\left(v \alpha_{k}^{2}-\lambda_{k}^{2}\right) \frac{\alpha_{k} \cosh \tilde{\alpha}_{k}}{\alpha_{k}^{2}+\mu_{s}^{2}}+B_{k}\left(v \beta_{k}^{2}-\lambda_{k}^{2}\right) \frac{\beta_{k} \cosh \tilde{\beta}_{k}}{\left.\beta_{k}^{2}+\mu_{s}^{2}\right]}\right]=0 \\
C_{s} \xi_{s}\left[\xi_{s}^{2}-(2-v) \mu_{s}^{2}+T_{y}\right]+D_{s} \eta_{s}\left[\eta_{s}^{2}-(2-v) \mu_{s}^{2}+T_{y}\right]=0 \\
A_{k}\left(\alpha_{k}^{2}-v \lambda_{k}^{2}\right) \sinh \tilde{\alpha}_{k}+B_{k}\left(\beta_{k}^{2}-v \lambda_{k}^{2}\right) \sinh \tilde{\beta}_{k} \\
+2 \sum_{s=1}^{\infty}\left[\frac{\mu_{s}^{2}-v \xi_{s}^{2}}{\lambda_{k}^{2}+\xi_{s}^{2}}\left\{C_{s}\left[(-1)^{\tilde{k}} \xi_{s}+\lambda_{k} \sinh \xi_{s}\right]-E_{s} \lambda_{k} \cosh \xi_{s}\right\}\right. \\
\left.+\frac{\mu_{s}^{2}-v \eta_{s}^{2}}{\lambda_{k}^{2}+\eta_{s}^{2}}\left\{D_{s}\left[(-1)^{\tilde{k}} \eta_{s}+\lambda_{k} \sinh \eta_{s}\right]-F_{s} \lambda_{k} \cosh \eta_{s}\right\}\right]=0 \\
A_{k} \alpha_{k}\left[\alpha_{k}^{2}-(2-v) \lambda_{k}^{2}\right] \cosh \tilde{\alpha}_{k}+B_{k} \beta_{k}\left[\beta_{k}^{2}-(2-v) \lambda_{k}^{2}\right] \cosh \tilde{\beta}_{k}=0 .
\end{gathered}
$$

Here, decomposition of hyperbolic functions of variable $x$ into Fourier series by sines was used:

$$
\begin{aligned}
& \sinh \alpha_{k} x=-\frac{4 \alpha_{k}}{\gamma} \cosh \tilde{\alpha}_{k} \sum_{s=1,3, \ldots}^{\infty} \frac{(-1)^{\tilde{s}}}{\alpha_{k}^{2}+\mu_{s}^{2}} \sin \mu_{s} x \\
& \sinh \beta_{k} x=-\frac{4 \beta_{k}}{\gamma} \cosh \tilde{\beta}_{k} \sum_{s=1,3, \ldots}^{\infty} \frac{(-1)^{\tilde{s}}}{\beta_{k}^{2}+\mu_{s}^{2}} \sin \mu_{s} x .
\end{aligned}
$$

\section{Results}

The computational process of determining critical loads and forms of equilibrium was organized according to the above algorithm in the Maple computer mathematics system. Poisson's ratio $v$ in all calculations was accepted equal to 0.3 . Parameters of the computational process: $T_{y}$ is the intensity value of the relative compressive load applied to edge $\mathrm{y}=1 ; \gamma=a / b$ is the plate side ratio; $N$ is the number of members in series, $N_{C}$ is the number of iterations. The coefficients of series (7), (8) or (34), (35) were printed out at each iteration, which allowed controlling the process of successive approximations, determining 
its convergence or divergence. The program provided for the construction of a 3D graph of the curved surface of the plate after finding the critical value. The series held 29 members. The larger number of members did not significantly affect the accuracy of calculations, but required an increase in the number of significant figures in the calculations (more than 400). Otherwise, the computational process was "locked" near the critical load, i.e. all values tended sharply to infinity. The number of iterations was 50 . The calculating time of each load variant was no more than two minutes. The strategy of the brute force search of load was dictated by the nature of the values of the required coefficients and did not take much time.

The obtained first three critical loads of symmetric forms and the first critical loads of antisymmetric forms of equilibrium for the plates with a side ratio of $1 / 4,1 / 2,1,2$, and 4 are shown in Table 1 and the corresponding 3D forms of equilibrium are shown in Figs. 221 .

Table 1. Critical loads $T_{\mathrm{y}}=\mathrm{T}_{\mathrm{Y}} \mathrm{b}^{2} / \mathrm{D}$ for a rectangular cantilever plate with different side ratios.

\begin{tabular}{|c|c|c|c|c|}
\hline \multicolumn{5}{|c|}{ Plate side ratio } \\
\hline$\gamma=1 / 4$ & $\gamma=1 / 2$ & $\gamma=1$ & $\gamma=2$ & $\gamma=4$ \\
\hline \multicolumn{5}{|c|}{ Symmetric solution } \\
\hline 2.0587 & 2.0724 & 2.1057 & 2.1623 & 2.2455 \\
18.936 & 19.59 & 20.457 & 20.814 & 21.568 \\
54.116 & 56.55 & 58.597 & 75.47 & 76.71 \\
\hline \multicolumn{5}{|c|}{ Antisymmetric solution } \\
\hline 122.95 & 29.36 & 8.08 & 3.884 & 2.853 \\
\hline
\end{tabular}

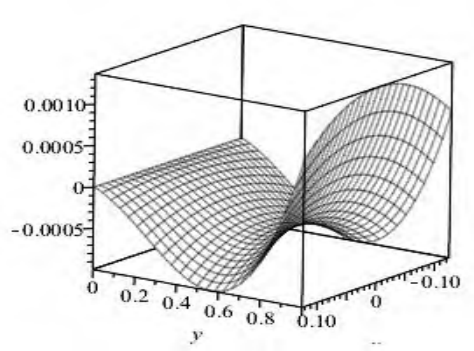

Fig. 2. First symmetric form of equilibrium of rectangular plate $\gamma=1 / 4$ at $\mathrm{T}_{\mathrm{CR} 1}=2.0587$.

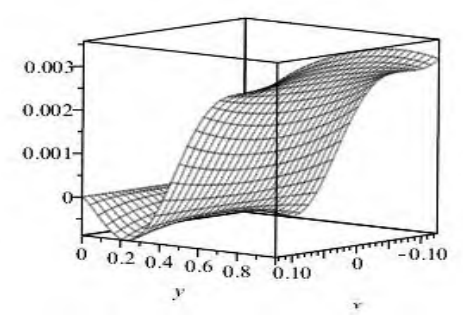

Fig. 4. Third symmetric form of equilibrium of rectangular plate $\gamma=1 / 4$ at $\mathrm{T}_{\mathrm{CR} 3}=54.116$.

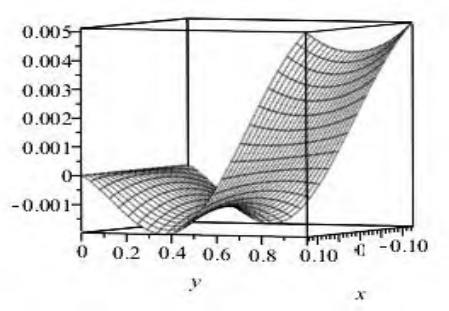

Fig. 3. Second symmetric form of equilibrium of rectangular plate $\gamma=1 / 4$ at $\mathrm{T}_{\mathrm{CR} 2}=18.936$.

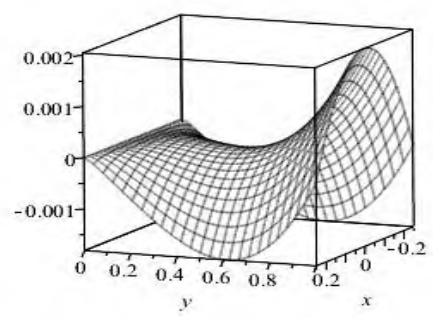

Fig. 5. First symmetric form of equilibrium of rectangular plate $\gamma=1 / 2$ at $\mathrm{T}_{\mathrm{CR} 1}=2.0724$. 


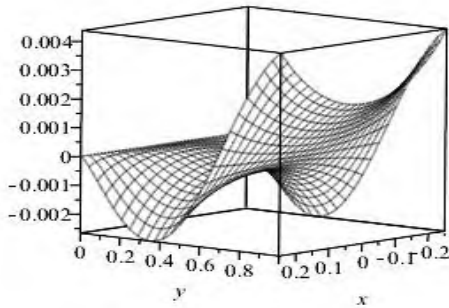

Fig. 6. Second symmetric form of equilibrium of rectangular plate $\gamma=1 / 2$ at $\mathrm{T}_{\mathrm{CR} 2}=19.59$.

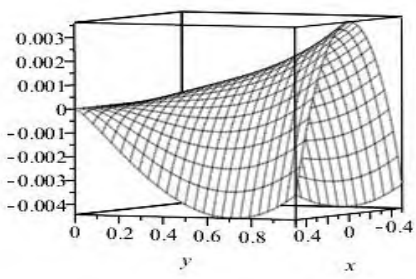

Fig. 8. First symmetric form of equilibrium of a square plate at $\mathrm{T}_{\mathrm{CR} 1}=2.1057$.

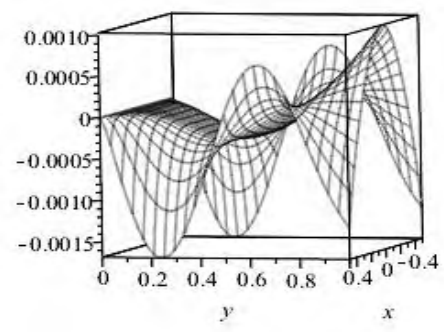

Fig. 10. Third symmetric form of equilibrium of a square plate at $\mathrm{T}_{\mathrm{CR} 3}=58.597$.

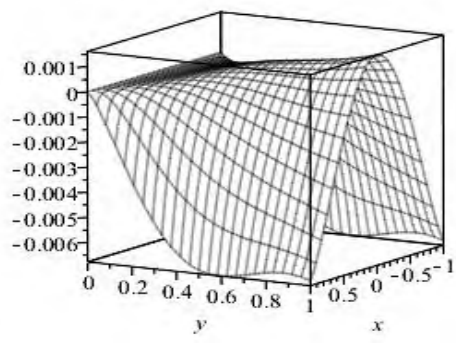

Fig. 12. Second symmetric form of equilibrium of rectangular plate $\gamma=2$ at TCR2 $=20.814$.

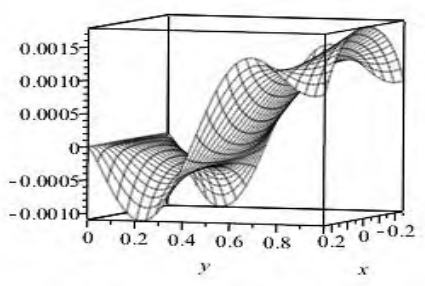

Fig. 7. Third symmetric form of equilibrium of rectangular plate $\gamma=1 / 2$ at $\mathrm{T}_{\mathrm{CR} 3}=56.55$.

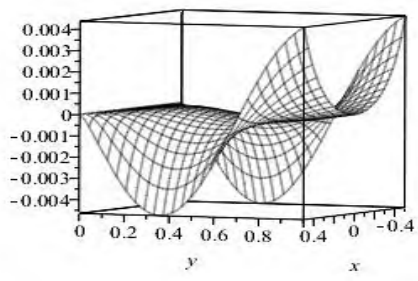

Fig. 9. Second symmetric form of equilibrium of a square plate at $\mathrm{T}_{\mathrm{CR} 2}=20.457$.

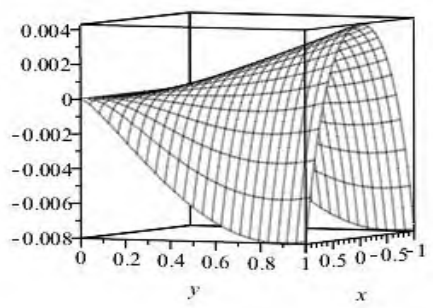

Fig. 11. First symmetric form of equilibrium of rectangular plate $\gamma=2$ at $\mathrm{T}_{\mathrm{CR} 1}=2.1623$.

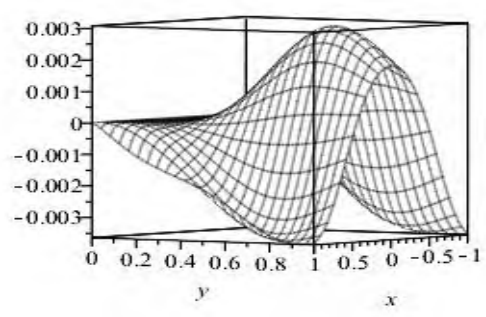

Fig. 13. Third symmetric form of equilibrium of rectangular plate $\gamma=2$ at $\mathrm{T}_{\mathrm{CR} 3}=75.47$. 


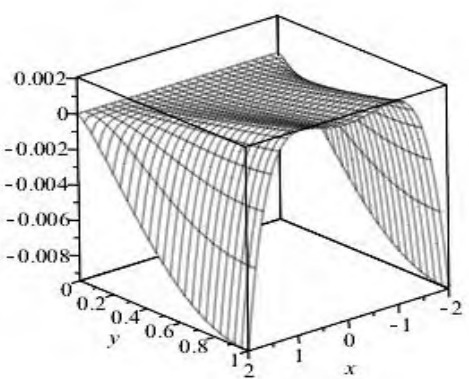

Fig. 14. First symmetric form of equilibrium of rectangular plate $\gamma=4$ at $\mathrm{T}_{\mathrm{CR} 1}=2.2455$.

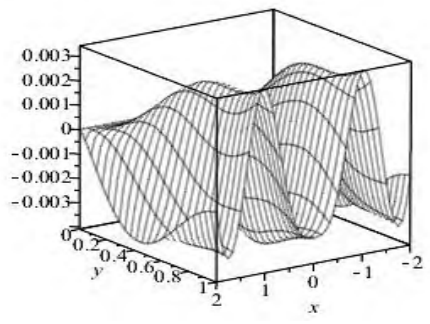

Fig. 16. Third symmetric form of equilibrium of rectangular plate $\gamma=4$ at $\mathrm{T}_{\mathrm{CR} 3}=76.71$.

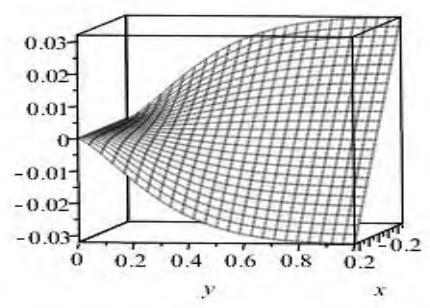

Fig. 18. First antisymmetric form of equilibrium of rectangular plate $\gamma=0.5$ at $\mathrm{T}_{\mathrm{CR} 1}=29.36$.

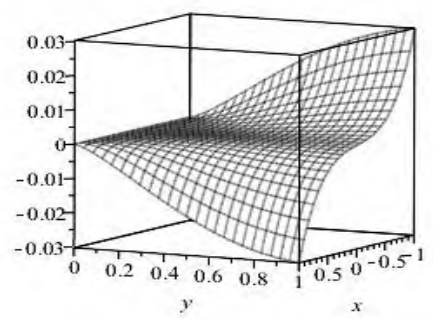

Fig. 20. First antisymmetric form of equilibrium of rectangular plate $\gamma=2$ at $\mathrm{T}_{\mathrm{CR} 1}=3.884$.

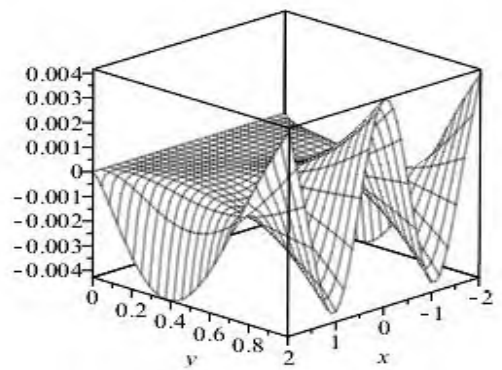

Fig. 15. Second symmetric form of equilibrium of rectangular plate $\gamma=4$ at $\mathrm{T}_{\mathrm{CR} 2}=21.568$.

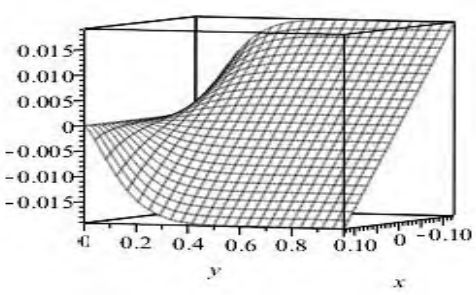

Fig. 17. First antisymmetric form of equilibrium of rectangular plate $\gamma=0.25$ at $\mathrm{T}_{\mathrm{CR} 1}=122.95$.

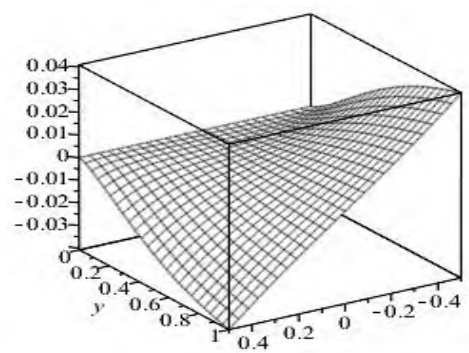

Fig. 19. First antisymmetric form of equilibrium of a square plate at $\mathrm{T}_{\mathrm{CR} 1}=8.08$.

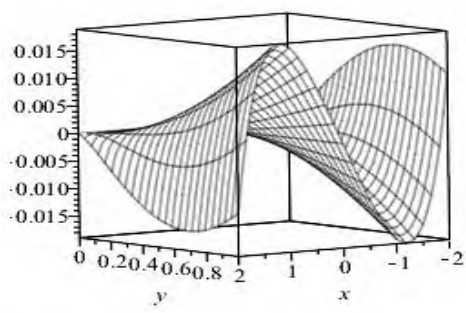

Fig. 21. First antisymmetric form of equilibrium of rectangular plate $\gamma=4$ at $\mathrm{T}_{\mathrm{CR} 1}=2.853$. 


\section{Discussion}

The obtained forms of equilibrium allow tracing their change depending on the change of the plate side ratio. Plates with a narrow clamped edge $(\gamma=1 / 4$ and less $)$ behave approximately like cantilever rods at the symmetric solution, and wide plates $(\gamma=4$ and more) at the first two critical loads have almost a flat form in the central part (symmetric solution), and on lateral free edges sharply change it. The third symmetric form of equilibrium of the wide plate $(\gamma=4)$ shows a sharp change in the direction perpendicular to the compressive load application. This shows a qualitative difference in the behavior of wide cantilever plates with an increasing load.

In paper [17], the first critical load for a square cantilever plate was determined when a uniform pressure had been applied to the free edge parallel to the clamped edge. This value ркр $=2.4571 \mathrm{D} / \mathrm{a} 2$ was obtained by the energy method from the condition that the second order determinant is equal to zero relative to compressive force $\mathrm{p}$. In this paper, the corresponding value is $2.1057 \mathrm{D} / \mathrm{a} 2$. These values are comparable, but the energy methods usually give excessive results, as in this case.

In paper [23], using the simplex method based on the nonlocal theory of elasticity of Eringen for nanoscale plates, numerical results were obtained for elastic cantilever plates with a relative thickness of 0.001 , i.e., super thin plates. Therefore, these results are difficult to compare with ours, although they are quite close, for example, for a plate with side ratio $\gamma=2$, the first critical loads are respectively 2.4174 [23] vs. 2.1623 (when converted to our designations); the second (symmetric form) critical loads are respectively 20.5173 [23] vs. 20.814 .

The proposed method of solving the problem of stability of a high-elastic rectangular cantilever plate allows determining the carrying capacity of this element with high accuracy, increasing the number of members in the series, the number of iterations, and the mantissa length during calculations.

\section{Conclusion}

The obtained results can find application in engineering design organizations to calculate stability of cantilever elements. They will also be in demand in nanoengineering when manufacturing nanosensors. The knowledge of critical loads will help to avoid destruction of these elements or to establish their behavior in supercritical areas. The found spectrum of critical loads can be used for constructions made of new materials of high elasticity.

With the developed method and computational algorithm, it is possible to find the specified loads for plates with any side ratio. The solution of the problem in a dimensionless form allows determining critical loads for thin plates of different thicknesses, modulus of elasticity and Poisson's ratio.

\section{References}

1. M.V. Sukhoterin, S.O. Baryshnikov, D.A. Aksenov, American Journal of Applied Sciences 13(12), 1442-1451 (2016)

2. L. Rui, W. Pengcheng, Y. Zekun, T. Linghui, Applied Mathematical Modelling 53(97) (2017)

3. R.A. Abdikarimov, V.M. Zhgutov, Magazine of Civil Engineering 24(6), 12-22 (2011)

4. V.V. Lalin, L.A. Rozin, D.A. Kushova, Magazine of Civil Engineering 36(1), 87-96 (2013) 
5. I.D. Ievzerov, Magazine of Civil Engineering 45(1), 6-11 (2014)

6. M.R. Eslami, Buckling and Postbuckling of Beams (Plates, and Shells, Springer Publishing, New York, 2018)

7. Yu.Ya. Tyukalov, Magazine of Civil Engineering 91(7), 80-97 (2019)

8. S. Kshirsagar, K. Bhaskar, Journal Sound and Vibration 314(3-5), 837-850 (2008)

9. A.A. Jafari, S.A. Eftekhari, Applied Mathevatics and Computanion 218(6), $2670-$ 2692 (2011)

10. G.C. Tsiatas, A.J. Yiotis, Engineering Analysis with Boundary Elements 37(3), 579584 (2013)

11. M.V. Sukhoterin, T.P. Knysh, L.V. Annenkov, Vestnik gosudarstvennogo universiteta morskogo i rechnogo flota im. admirala S.O. Makarova 18(2), 51-58 (2013)

12. M.V. Sukhoterin, E.V. Potekhina, L.V. Annenkov, Vestnik gosudarstvennogo universiteta morskogo i rechnogo flota im. admirala S.O. Makarova 24(2), 41-51 (2014)

13. L.V. Annenkov, Vestnik gosudarstvennogo universiteta morskogo i rechnogo flota im. admirala S.O. Makarova 31(3), 48-53 (2015)

14. M.V. Sukhoterin, S.O. Baryshnikov, T.P. Knysh, N.F. Pizhurina, MATEC Web of Conferences 239 (2018)

15. S.O. Papkov, J.R. Banerjee, Journal of Sound and Vibration 339, 342 - 359 (2015)

16. S.O. Papkov, O.A. Dovgalenko, and M.A. Chevin, Innovacii v nauke 66(5), 22-26 (2017)

17. C. Xiang-sheng, Applied Mathematics and Mechanics (English Edition, China) 8(7), 673-683 (1987)

18. C. Xiang-sheng, Applied Mathematics and Mechanics (English Edition, China) 11(4), 377-383 (1990)

19. C. Xiang-sheng, Applied Mathematics and Mechanics (English Edition, China) 9(8), 787-792 (1988)

20. Yu. Yi-wu, Applied Mathematics and Mechanics (English Edition, China) 14, 151-155 (1993)

21. L. Jiang, S. Wu, H. Zheng, Advanced Materials Research 671-674, 1596-1599 (2013)

22. T.N. Isaulova, I.M. Lavit, Journal of Applied Mechanics and Technical Physics 52(4), 664-675 ( 2011)

23. W. Wang, D. Rong, X. Chenghui, Ju. Zhang, X. Xu, Z. Zhon, Journal of Vibration Engineering \& Technologies 58(5) (2019)

24. A.C. Eringen, Nonlocal Continuum Field Theories (New York, Springer, 2002)

25. S. Timoshenko, S. Woinowsky-Krieger, Theory of Plates and Shells (McGraw-Hill Book Company, New York, Toronto, London, 1959) 